\title{
Point-of-care testing for influenza in a university emergency department (ED): A prospective study
}

\author{
Benjamin Perlitz ${ }^{1}$, Anna Slagman ${ }^{1}$, Jennifer Hitzek ${ }^{1}$, Dorothee Riedlinger ${ }^{1}$, and Martin \\ Möckel ${ }^{1}$ \\ ${ }^{1}$ Charite Universitatsmedizin Berlin
}

March 14, 2021

\begin{abstract}
Background: Seasonal influenza is a burden for emergency departments. The aim of this study was to investigate whether point-of-care (POC) PCR testing can be used to reduce staff sick days and improve diagnostic and therapeutic procedures.

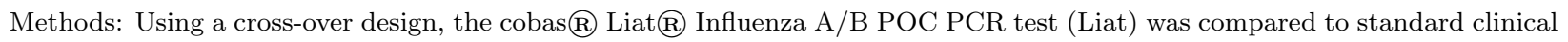
practice during the 2019/2020 influenza season. All adult patients (aged [?]18 years) with fever ([?]38degC) and respiratory symptoms were included. Primary endpoints were prevalence of influenza infections in the ED and staff sick days. Secondary endpoints were frequency of antiviral and antibacterial therapy, time between admission and test result or treatment initiation, patient disposition, ED length of stay (LOS) and for in-patients mortality and LOS. Nurses were interviewed about handling and integration of POC testing. The occurrence of SARS-CoV-2 infections coincided with the second half of the study. Results: A total of 828 patients were enrolled in the study. All 375 patients of the intervention group were tested with Liat, 103 of them $(27.6 \%)$ tested positive. During the intervention period staff sick days were reduced by $34.4 \%$ ( $\mathrm{p}=0.023)$. Significantly more patients in the intervention group received antiviral therapy with neuraminidase-inhibitors $(7.2 \%$ vs. $3.8 \%, p=0.028)$ and tested patients received antibiotics more frequently $(40.0 \%$ vs. $31.6 \%, \mathrm{p}=0.033)$. Patients with POC test were transferred to external hospitals significantly more often $(5.6 \%$ vs. $1.3 \%, \mathrm{p}=0.01)$. Conclusion: We conclude that POC testing for influenza is useful in the ED, especially if it is heavily frequented by patients with respiratory symptoms.
\end{abstract}

\section{Hosted file}

Point-of-care testing for influenza in a university emergency department A prospective study_Final.pdf available at https://authorea.com/users/401447/articles/513514-point-of-care-testing-forinfluenza-in-a-university-emergency-department-ed-a-prospective-study 